\title{
Induction of plasticity in the human motor cortex by pairing an auditory stimulus with TMS
}

\author{
Paul F. Sowman ${ }^{1,2,3}$ *, Søren S. Dueholm ${ }^{1,4}$, Jesper H. Rasmussen ${ }^{1,4}$ and Natalie Mrachacz-Kersting ${ }^{4}$ \\ ${ }^{1}$ Department of Cognitive Science, Macquarie University, Sydney, NSW, Australia \\ ${ }^{2}$ Perception and Action Research Centre (PARC), Faculty of Human Sciences, Macquarie University, Sydney, NSW, Australia \\ ${ }^{3}$ Australian Research Council Centre of Excellence in Cognition and its Disorders (CCD), Macquarie University, Sydney, NSW, Australia \\ ${ }^{4}$ Department of Health Science and Technology, Center for Sensory-Motor Interaction (SMI), Aalborg University, Aalborg, Denmark
}

\section{Edited by:}

Carolyn McGettigan, Royal

Holloway University of London, UK

Reviewed by:

Alessandro D'Ausilio, Italian Institute of Technology, Italy

Dan Kennedy-Higgins, University

College London-Speech, Hearing

and Phonetic Sciences, UK

*Correspondence:

Paul F. Sowman, Department of

Cognitive Science, Level 3

Australian Hearing Hub, 16

University Drive, Macquarie

University, Sydney, NSW 2109,

Australia

e-mail:paul.sowman@mq.edu.au
Acoustic stimuli can cause a transient increase in the excitability of the motor cortex. The current study leverages this phenomenon to develop a method for testing the integrity of auditorimotor integration and the capacity for auditorimotor plasticity. We demonstrate that appropriately timed transcranial magnetic stimulation (TMS) of the hand area, paired with auditorily mediated excitation of the motor cortex, induces an enhancement of motor cortex excitability that lasts beyond the time of stimulation. This result demonstrates for the first time that paired associative stimulation (PAS)-induced plasticity within the motor cortex is applicable with auditory stimuli. We propose that the method developed here might provide a useful tool for future studies that measure auditory-motor connectivity in communication disorders.

Keywords: paired associative stimulation, transcranial magnetic stimulation, auditory motor integration, speech sounds, plasticity, motor cortex, auditory cortex

\section{INTRODUCTION}

Paired associative stimulation (PAS) is a technique used to experimentally induce long-lasting changes in cortical excitability (Stefan et al., 2002; Ridding and Flavel, 2006; MrachaczKersting et al., 2007; Murakami et al., 2008; Kumpulainen et al., 2012). Most commonly in PAS studies, electrical stimulation of the median nerve is paired with transcranial magnetic stimulation (TMS) of the contralateral motor cortex. The nerve impulse resulting from the somatosensory stimulus can be timed to arrive at the cortical level milliseconds prior the TMS pulse in order to induce a long-lasting increase in excitability - a process that is thought to be mediated by a Hebbian long-term potentiation (LTP)-like process (Stefan et al., 2002).

In recent years, modified PAS protocols have been designed that apply more ecologically valid stimuli in place of either the TMS or the electrical somatosensory stimulation, e.g., TMS paired with movement (Thabit et al., 2010) or electrical somatosensory stimulation paired with motor imagery (Mrachacz-Kersting et al., 2012). PAS protocols have also moved beyond ubiquitous sensorimotor associations to demonstrate that pairing a TMS-induced cortical activation outside the motor cortex with a homotopic sensory activation can induce enhanced responses to sensory inputs. For example, Schecklmann et al. (2011) showed that pairing a TMS pulse to the auditory cortex with a simple tone could induce a prolonged decrement of the auditory evoked potential. Cortical stimulation has also been paired with visual stimuli to demonstrate the capacity for visuomotor integration to mediate plastic changes in motor cortex (Suppa et al., 2013). To date however, the connections known to exist between the auditory and motor domains have not been tested for their capacity to induce motor cortex plasticity.

A number of well-described functional links between audition and the motor system exist. These range from protective reflexive motor activations in response to signals of potential danger (Forbes and Sherrington, 1914) to the complex feedback and feedforward communication necessary for fluent speech to occur (Tourville et al., 2008; Perkell, 2012). These connections allow us to, for example, modulate the volume of our speech to appropriately match the ambient environmental noise (Lane and Tranel, 1971) or modulate the sensitivity of our sensory system to compensate for speech-induced reafference (Curio et al., 2000).

Motoric activation via auditory inputs has been demonstrated in a number of experiments that have used TMS to probe the link between speech perception and motor representations. Modulation of motor cortical excitability during speech perception has been demonstrated to occur in the cortical representations of the hand (Flöel et al., 2003), lips (Watkins et al., 2003) and tongue (Fadiga et al., 2002; Roy et al., 2008).

Despite evidence suggesting a strong connection between auditory and motor centers, auditory stimuli have not yet been used in a modified PAS study to induce plasticity in the motor area. The aim of the current study was to investigate whether it is possible to induce plasticity in the motor system by pairing auditory stimuli and TMS. The development of such a protocol would in future allow for the direct investigation of auditorimotor 
linkages in a number of disorders where these are thought to be abnormal. Auditorimotor disconnection or dysfunction has, for example, been proposed to underpin the speech dysfluencies that characterize stuttering (Neef et al., 2011) and the misattribution of self-produced speech that may produce auditory hallucinations in schizophrenia (Ford et al., 2005).

\section{METHODS}

Two separate experiments (A and B) were conducted. Given that the timing of stimuli in a PAS protocol is critical for facilitating plastic change (Stefan et al., 2002; Wolters et al., 2005; MrachaczKersting et al., 2007; Murakami et al., 2008; Kumpulainen et al., 2012), the aim of Experiment A was to find, at a group level, the optimal offset timing of the motor cortical excitation from the onset of the auditory stimulus. This offset was determined by applying TMS pulses at different latencies relative to the onset of the auditory stimulus and measuring the conditioned motor evoked potential (MEP) in the right first dorsal interosseus (FDI) muscle.

The PAS protocol implemented in Experiment B was informed by the results of Experiment A. First a baseline session was conducted where MEPs (TMS with no auditory stimuli) were collected and saved as pre-PAS measurements. This was followed by an intervention block which consisted of the auditorimotor PAS-protocol. During the intervention block, subjects received an auditory stimulus paired with TMS using the optimal time latency between stimulations that was found in Experiment A. After the intervention session, post-PAS MEPs were recorded immediately after and then 15 min after the session ended (post and post15, respectively). By comparing pre- with post-MEPs and post15MEPs it was possible to evaluate whether motor cortex excitability changes had occurred, how fast they evolved and whether they were long-lasting.

\section{EXPERIMENT A-TIMING OF STIMULI SUBJECTS}

Experiment A was performed on 12 healthy right-handed volunteers (9 males), aged 18-36 years (mean $24.2 \pm 5.0$ years). Prior to commencement of the experiment subjects completed a standard TMS screening questionnaire and provided written informed consent. None of the subjects reported any history of hearing impairment, neurological disease or mental illness, was taking regular medication or had a history brain injury. This study was reviewed and approved by the Human Research Ethics Committee of Macquarie University.

\section{EXPERIMENTAL PROCEDURE}

Subjects were seated in a chair with their right arm and hand resting in a comfortable position on an armrest. An armrest was used in order to eliminate hand movements during recordings. During the experiment the subject was told to relax, avoid any movement of the right arm and hand and to have their eyes open. Surface EMG $($ sEMG $)$ was recorded $(1000 \times$ gain, bandpass filtered from $20-500 \mathrm{~Hz}$ ) from a bipolar electrode (Medi-Trace 100, Kendall/Tyco Healthcare, USA) montage. One electrode was placed over the muscle belly of the right FDI muscle and the other electrode was placed over the proximal metacarpal of the index finger.

A monophasic transcranial magnetic stimulator (Magstim model 200, Magstim, Whitland, UK), with a focal figure-of-eight stimulating coil (90-mm outer diameter), was used to elicit MEPs from the right FDI muscle. The stimulating coil was held tangentially to the skull with the coil oriented $45^{\circ}$ to the parasagittal plane and the handle pointing laterally and posteriorly. The center of the coil junction was placed over the primary motor cortex (M1) hand area of the left hemisphere and the "motor hot spot" was determined as the site where TMS consistently elicited the largest MEPs.

Resting motor threshold (MT) was determined by finding the lowest stimulation intensity of the motor hotspot for the right FDI needed in order to obtain an MEP with a peak-to-peak amplitude of $50 \mu \mathrm{V}$ in 5 out of 10 consecutive stimulations. The TMS test intensity was then set at $120 \%$ of resting MT. Eight different TMS conditions were tested. These consisted of seven auditory-stimulation/TMS pairs and one TMS condition without associated auditory stimulation (baseline). The auditorystimulation/TMS pairs consisted of a test TMS pulse applied at one of seven different intervals $(25,50,100,150,200,250$ and $300 \mathrm{~ms}$ ) after the onset of the auditory stimulus. The auditory stimulus consisted of a male voice pronouncing the word "Hey!" played back at $80 \mathrm{~dB}$ SPL via Etymotic ER-1 insert tube-phones. We chose to use a speech sounds because previous research suggests that speech sounds strongly activate the motor cortex e.g., Flöel et al. (2003). However, other evidence suggests that the motor cortex might be also activated by non speech sounds (Watkins et al., 2003; Alibiglou and Mackinnon, 2012) so we also included a condition in which the auditory stimulus matched the amplitude envelope of the speech stimulus but consisted entirely of white noise (Pulvermüller et al., 2006). This signal-correlated noise (SCN) stimulus was created using Praat (Boersma and Weenink, 2013). Time and frequency domain comparisons of the two signals are displayed in Figure $\mathbf{1}$.

The order of all seven auditory-stimulation/TMS pairs and stimulus types (speech or SCN) was randomly intermingled and presented with an intertrial interval (ITI) that randomly varied between 4000 and $5000 \mathrm{~ms}$ in two blocks such that the total number of stimuli per condition was 16 . The total number of trials was hence 128 ( 16 baseline trials $+7 \times 16$ conditioned trials). The duration of the experiment was approximately $25 \mathrm{~min}$.

\section{DATA PROCESSING}

Offline MEP analysis was conducted using a custom MATLAB (The Mathworks, USA) script. The average MEP amplitude calculated for each sound type and auditory-stimulation/TMS pair was expressed as a function of the average pre MEP (baseline).

\section{STATISTICAL ANALYSIS}

A repeated measures ANOVA with the factors delay (auditorystimulus/TMS interval) and condition (speech or SCN) was performed on the averaged MEPs. A two-tailed, one-sample $t$ test was then used to determine the time points at which the conditioned MEPs differed significantly from baseline using an $\alpha$-value of 0.05 . 

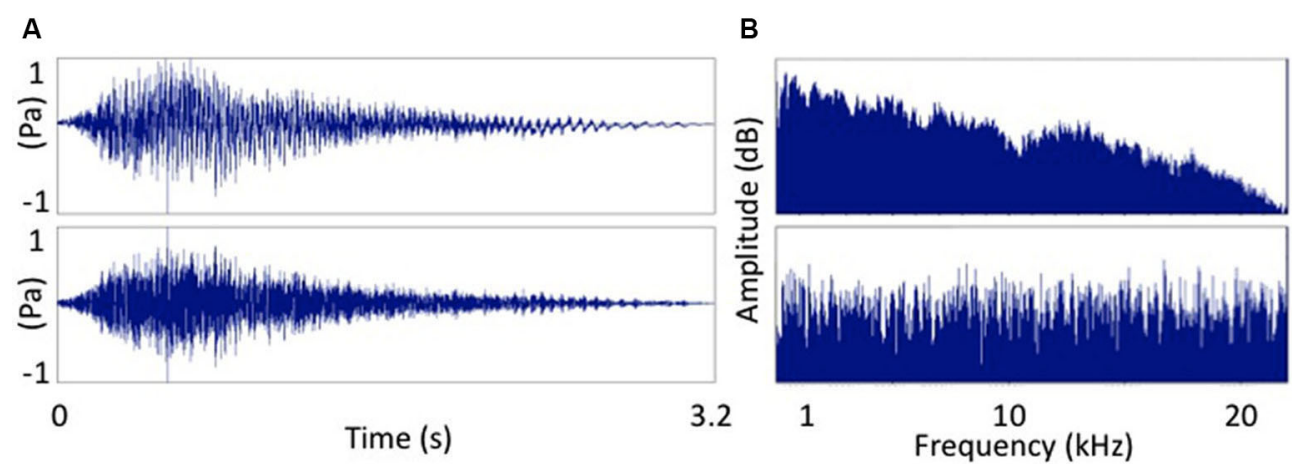

FIGURE 1 | Two sounds used as auditory stimuli. (A) The word "Hey!" and (B) signal correlated noise version of (A). Frequency spectra of the two

auditory stimuli. (A) The word "Hey!" and (B) signal correlated noise (white noise) version of (A).
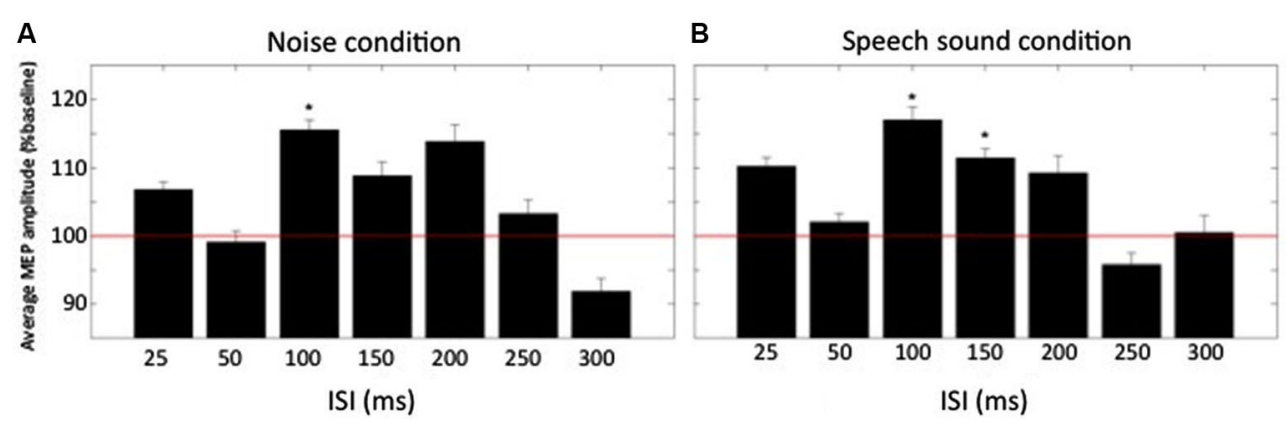

FIGURE 2 | Normalized averaged MEP amplitudes (+SEM) at different times relative to the conditioning auditory stimulus. (A) MEP amplitudes for the condition "noise" for all ISIs $(n=12)$. (B) MEP amplitudes for the condition "speech sound". Baseline is represented by the red horizontal line. * denotes average amplitude significantly different from baseline $(p<0.05)$.

\section{EXPERIMENT B-AUDITORIMOTOR PAS SUBJECTS}

Experiment $\mathrm{B}$ was performed on 10 healthy right-handed volunteers ( 8 males), aged $18-31$ years (mean $24.5 \pm 3.3$ years) without any prior neurological medical history. Written informed consent was obtained from each subject before participation in the study.

\section{EXPERIMENTAL PROCEDURE}

The procedure used in Experiment $\mathrm{B}$ was similar to the one used in Experiment A. The main difference was that a single auditory stimulus/TMS interval $(100 \mathrm{~ms})$ was used during the PAS induction period in Experiment B. As no difference in MEP facilitation between the speech and SCN stimulus conditions was found in Experiment A we arbitrarily chose to use only the speech stimulus in Experiment B. PAS induction following baseline MEP recording consisted of a total of 200 auditory stimulus/TMS pairs applied with a 4000 $5000 \mathrm{~ms}$ random interval between each pair. A 2 min pause in stimulation after 100 pairs were applied was included. The total duration of the experiment was approximately $27 \mathrm{~min}$ (introduction: $10 \mathrm{~min}$, part one: $7.5 \mathrm{~min}$, pause: $2 \mathrm{~min}$, part two: $7.5 \mathrm{~min})$.

\section{STATISTICAL ANALYSIS}

A two-tailed, one-sample $t$-test was used to determine significant differences between pre-MEPs (baseline), post-MEPs and post15MEPs using an $\alpha$-value of 0.05 .

\section{RESULTS}

Mean $( \pm$ SEM) MEP threshold in Experiment A was $45.5 \pm 2.1 \%$ of stimulator output and $46.6 \pm 2.4 \%$ in Experiment B.

Results from Experiment A are shown in Figure 2. A repeated measures ANOVA showed that there was a significant effect of delay on the size of the MEP $F_{(6,66)}=2.3, p=0.045$. There was no significant effect of condition nor significant interaction between delay and condition. Within condition comparison of mean normalized MEPs to baseline by means of a two-tailed one-sample $t$-test revealed that in the noise condition, MEPs were significantly increased above baseline for one ISI: $100 \mathrm{~ms}$ $\left(115.5 \pm 5.2 \%\right.$ of baseline, $\left.t_{(11)}=3.0, p=0.012\right)$. For the speech sound condition two ISIs had MEPs that were significantly increased above baseline: $\mathrm{ISI}=100 \mathrm{~ms}(117.0 \pm 6.5 \%$ of baseline, $\left.t_{(11)}=2.6, p=0.023\right)$ and ISI $=150 \mathrm{~ms}(111.4 \pm 4.7 \%$ of baseline, $\left.t_{(11)}=2.4, p=0.035\right)$.

Results from Experiment B show that across all subjects the averaged MEP peak-to-peak amplitude increased to $148 \%$ (post) 


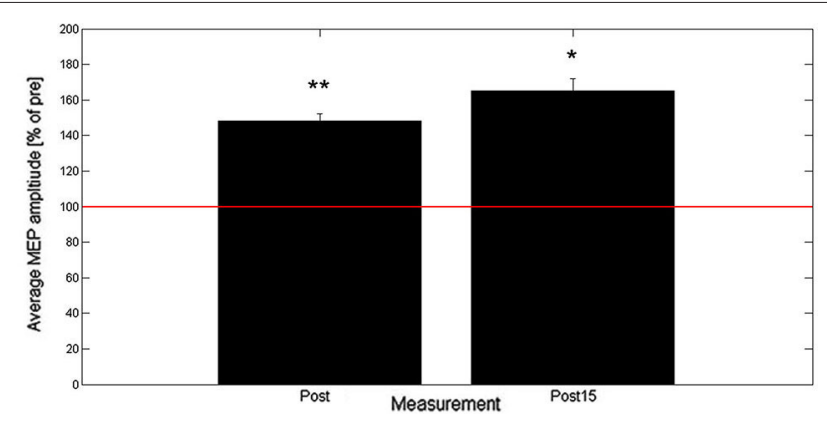

FIGURE 3 | Averaged post- and post15-MEP-amplitudes (+SEM) as percentage of baseline $(\boldsymbol{n}=\mathbf{1 0})$. Baseline is represented by the red horizontal line. ${ }^{*}$ denotes average amplitude significantly different from baseline $\left(^{*}<0.05,{ }^{*}<0.01\right)$.

and 165\% (post15) of baseline as shown in Figure 3. Two-tailed one-sample $t$-tests showed a significant increase in normalized MEP peak-to-peak amplitude for post $\left(t_{(9)}=3.8, p=0.004\right)$ and post15 $\left(t_{(9)}=2.9, p=0.018\right)$. Comparison between post and post 15 by means of a paired $t$-test revealed no significant difference $\left(t_{(9)}=1.06, p=0.32\right)$.

\section{DISCUSSION}

The current study demonstrates for the first time that long-lasting motor cortical plasticity can be induced by an auditorimotor PAS paradigm. This result is significant because it not only provides a new method for investigating auditorimotor integration, but importantly, also a method to directly probe the brain's capacity for auditorimotor plasticity.

We utilized a two-stage approach in developing this PAS paradigm. First, we identified the optimal ISI for eliciting an enhanced MEP response compared to baseline. This paradigm follows the empirical approach developed by Mrachacz-Kersting et al. (2007) to investigate PAS induced plasticity in the cortical representation of tibialis anterior. The optimal interval we found fits well with the temporal structure of the auditory N1 to speech sounds which peaks $100 \mathrm{~ms}$ after stimulus onset e.g., Liotti et al. (2010), and agrees with the TMS findings of Fadiga et al. (2002) and those of Roy et al. (2008), who found "phonological motor resonance" was present at $100 \mathrm{~ms}$ after their target speech sound stimulus onsets. In both studies the authors applied TMS to the tongue motor representation following the presentation of pseudo-words containing double consonants. The MEP response that they recorded in the tongue peaked in amplitude when the auditory stimulus to TMS interval was $100 \mathrm{~ms}$.

While we used a speech stimulus in these experiments, the lack of difference between the response to the speech stimulus and SCN found in Experiment A suggests that under the experimental conditions we have imposed, i.e., a repetitive presentation of a speech sound without the requirement for engagement on the part of the subject, the stimulus may not be processed as speech per se and should rather be considered a non-specific acoustic stimulus. This fact may explain why our results differ in part to those of Watkins et al. (2003) and Murakami et al. (2011) whose findings suggest that auditory-induced motor modulations related to speech listening are confined to the cortical representations of those muscles involved in articulation. Indeed, there is now a significant body of evidence to support the somatotopic arrangement of speech gesture perception (Fadiga et al., 2002; Roy et al., 2008; D’Ausilio et al., 2009, 2011; Möttönen and Watkins, 2009; Sato et al., 2010) but such findings do not necessarily rule out the non-specific motor activations in response to both speech and non-speech acoustic stimuli that have been documented using both TMS and other methods (Flöel et al., 2003; Alibiglou and Mackinnon, 2012; Fujioka et al., 2012).

The current study shows that repeated pairing of an acoustic stimulus with a TMS pulse to the motor cortex representation of the hand leads to a rapidly-evolving, long-lasting increase in cortical excitability. This effect was induced with an ISI of $100 \mathrm{~ms}$, a time interval that corresponded to the point of peak enhancement in the acoustic stimulus-conditioned MEP. Given that this ISI was converged upon using a method that used discrete intervals with a minimum step of $50 \mathrm{~ms}$, it is expected that this PAS technique could be refined further by reexamining the optimal sound-to-TMS interval using smaller time steps (i.e., less than $25 \mathrm{~ms}$ ) centered around $100 \mathrm{~ms}$. Moreover, using auditory evoked potentials to discover individualized N1 latencies, and then using these as the basis for the PAS ISI would likely refine the technique further. Since we were able to find a significant PAS effect in this proof of concept study, we posit that auditorimotor PAS is a robust effect that will provide a powerful tool for studying auditorimotor plasticity in the future.

Auditorimotor plasticity i.e., the capacity for strengthening of auditorimotor connections within the brain is essential for the acquisition of speech and the learning of musical competence. For this reason, techniques that can probe the brain's capacity for auditorimotor plasticity provide the opportunity to investigate some of the hypothesized mechanisms of conditions such as stuttering and specific language impairment (SLI) in which disordered motor learning has been documented (Namasivayam and van Lieshout, 2008; Mayor-Dubois et al., 2014). Both of those conditions have been associated with disordered sensorimotor integration (Hill, 2001; Neef et al., 2011; Cai et al., 2012, 2014) and, in the case of SLI, with disordered auditorimotor plasticity (Kurt et al., 2012). Additionally, disorders such as schizophrenia and tinnitus have been associated with disrupted auditorimotor connections (Cacace, 2003; Ford et al., 2005; Langguth et al., 2005) and synaptic plasticity (Møller, 2003; Stephan et al., 2009); the technique described herein is therefore a novel means to assess these associations. Beyond mechanistic investigation of disorders, associative stimulation using TMS has also been proposed as a therapeutic modality (Uy et al., 2003; Jayaram and Stinear, 2008; Michou et al., 2013). If it is established that disorders such as those described above involve a form of auditorimotor disconnection, then auditorimotor PAS could be used as a novel adjuvant therapy to assist in the re/establishment of appropriate sensorimotor mappings.

\section{ACKNOWLEDGMENTS}

Paul F. Sowman is supported by the National Health and Medical Research Council, Australia (\#543438, \#1003760) and the 
Australian Research Council (DE130100868). The authors would like to thank AC Etchell for his comments on the manuscript.

\section{REFERENCES}

Alibiglou, L., and Mackinnon, C. D. (2012). The early release of planned movement by acoustic startle can be delayed by transcranial magnetic stimulation over the motor cortex. J. Physiol. 590, 919-936. doi: 10.1113/jphysiol.2011.219592

Boersma, P., and Weenink, D. (2013). Praat: Doing Phonetics by Computer. [Computer program] (Version 5.3.51), retrieved 2 June 2013 from http://www.praat.org/

Cacace, A. T. (2003). Expanding the biological basis of tinnitus: crossmodal origins and the role of neuroplasticity. Hear. Res. 175, 112-132. doi: 10.1016/s03785955(02)00717-7

Cai, S., Beal, D. S., Ghosh, S. S., Guenther, F. H., and Perkell, J. S. (2014). Impaired timing adjustments in response to time-varying auditory perturbation during connected speech production in persons who stutter. Brain Lang. 129, 24-29. doi: 10.1016/j.bandl.2014.01.002

Cai, S., Beal, D. S., Ghosh, S. S., Tiede, M. K., Guenther, F. H., and Perkell, J. S. (2012). Weak responses to auditory feedback perturbation during articulation in persons who stutter: evidence for abnormal auditory-motor transformation. PLoS One 7:e41830. doi: 10.1371/journal.pone.0041830

Curio, G., Neuloh, G., Numminen, J., Jousmaki, V., and Hari, R. (2000). Speaking modifies voice-evoked activity in the human auditory cortex. Hum. Brain Mapp. 9, 183-191. doi: 10.1002/(sici)1097-0193(200004)9:4<183::aid-hbml>3. $0 . \mathrm{co} ; 2-\mathrm{Z}$

D’Ausilio, A., Bufalari, I., Salmas, P., Busan, P., and Fadiga, L. (2011). Vocal pitch discrimination in the motor system. Brain Lang. 118, 9-14. doi: 10.1016/j.bandl. 2011.02.007

D’Ausilio, A., Pulvermüller, F., Salmas, P., Bufalari, I., Begliomini, C., and Fadiga, L. (2009). The motor somatotopy of speech perception. Curr. Biol. 19, 381-385. doi: 10.1016/j.cub.2009.01.017

Fadiga, L., Craighero, L., Buccino, G., and Rizzolatti, G. (2002). Speech listening specifically modulates the excitability of tongue muscles: a TMS study. Eur. J. Neurosci. 15, 399-402. doi: 10.1046/j.0953-816x.2001.01874.x

Flöel, A., Ellger, T., Breitenstein, C., and Knecht, S. (2003). Language perception activates the hand motor cortex: implications for motor theories of speech perception. Eur. J. Neurosci. 18, 704-708. doi: 10.1046/j.1460-9568.2003. 02774.x

Forbes, A., and Sherrington, C. (1914). Acoustic reflexes in the decerebrate cat. Amer. J. Physiol. 35, 367-376.

Ford, J. M., Gray, M., Faustman, W. O., Heinks, T. H., and Mathalon, D. H. (2005). Reduced gamma-band coherence to distorted feedback during speech when what you say is not what you hear. Int. J. Psychophysiol. 57, 143-150. doi: 10. 1016/j.ijpsycho.2005.03.002

Fujioka, T., Trainor, L. J., Large, E. W., and Ross, B. (2012). Internalized timing of isochronous sounds is represented in neuromagnetic beta oscillations. J. Neurosci. 32, 1791-1802. doi: 10.1523/JNEUROSCI.4107-11.2012

Hill, E. L. (2001). Non-specific nature of specific language impairment: a review of the literature with regard to concomitant motor impairments. Int. J. Lang. Commun. Disord. 36, 149-171. doi: 10.1080/13682820118418

Jayaram, G., and Stinear, J. W. (2008). Contralesional paired associative stimulation increases paretic lower limb motor excitability post-stroke. Exp. Brain Res. 185, 563-570. doi: 10.1007/s00221-007-1183-x

Kumpulainen, S., Mrachacz-Kersting, N., Peltonen, J., Voigt, M., and Avela, J. (2012). The optimal interstimulus interval and repeatability of paired associative stimulation when the soleus muscle is targeted. Exp. Brain Res. 221, 241-249. doi: 10.1007/s00221-012-3165-x

Kurt, S., Fisher, S. E., and Ehret, G. (2012). Foxp2 mutations impair auditory-motor association learning. PLoS One 7:e33130. doi: 10.1371/journal.pone.0033130

Lane, H., and Tranel, B. (1971). The lombard sign and the role of hearing in speech. J. Speech Lang. Hear. Res. 14, 677-709.

Langguth, B., Eichhammer, P., Zowe, M., Kleinjung, T., Jacob, P., Binder, H., et al. (2005). Altered motor cortex excitability in tinnitus patients: a hint at crossmodal plasticity. Neurosci. Lett. 380, 326-329. doi: 10.1016/j.neulet.2005. 01.069

Liotti, M., Ingham, J. C., Takai, O., Paskos, D. K., Perez, R., and Ingham, R. J. (2010). Spatiotemporal dynamics of speech sound perception in chronic developmental stuttering. Brain Lang. 115, 141-147. doi: 10.1016/j.bandl.2010.07.007
Mayor-Dubois, C., Zesiger, P., Van der Linden, M., and Roulet-Perez, E. (2014). Nondeclarative learning in children with specific language impairment: predicting regularities in the visuomotor, phonological and cognitive domains. Child Neuropsychol. 20, 14-22. doi: 10.1080/09297049.2012. 734293

Michou, E., Mistry, S., Rothwell, J., and Hamdy, S. (2013). Priming pharyngeal motor cortex by repeated paired associative stimulation: implications for dysphagia neurorehabilitation. Neurorehabil. Neural Repair 27, 355-362. doi: 10. $1177 / 1545968312469837$

Møller, A. R. (2003). Pathophysiology of tinnitus. Otolaryngol. Clin. North Am. 36, 249-266, v-vi. doi: 10.1016/S0030-6665(02)00170-6

Möttönen, R., and Watkins, K. E. (2009). Motor representations of articulators contribute to categorical perception of speech sounds. J. Neurosci. 29, 98199825. doi: 10.1523/JNEUROSCI.6018-08.2009

Mrachacz-Kersting, N., Fong, M., Murphy, B. A., and Sinkjaer, T. (2007). Changes in excitability of the cortical projections to the human tibialis anterior after paired associative stimulation. J. Neurophysiol. 97, 1951-1958. doi: 10.1152/jn. 01176.2006

Mrachacz-Kersting, N., Kristensen, S. R., Niazi, I. K., and Farina, D. (2012). Precise temporal association between cortical potentials evoked by motor imagination and afference induces cortical plasticity. J. Physiol. 590, 1669-1682. doi: 10. 1113/jphysiol.2011.222851

Murakami, T., Restle, J., and Ziemann, U. (2011). Observation-execution matching and action inhibition in human primary motor cortex during viewing of speechrelated lip movements or listening to speech. Neuropsychologia 49, 2045-2054. doi: 10.1016/j.neuropsychologia.2011.03.034

Murakami, T., Sakuma, K., Nomura, T., Uemura, Y., Hashimoto, I., and Nakashima, K. (2008). Changes in somatosensory-evoked potentials and high-frequency oscillations after paired-associative stimulation. Exp. Brain Res. 184, 339-347. doi: 10.1007/s00221-007-1103-0

Namasivayam, A. K., and van Lieshout, P. (2008). Investigating speech motor practice and learning in people who stutter. J. Fluency Disord. 33, 32-51. doi: 10.1016/j.jfludis.2007.11.005

Neef, N. E., Jung, K., Rothkegel, H., Pollok, B., von Gudenberg, A. W., Paulus, W., et al. (2011). Right-shift for non-speech motor processing in adults who stutter. Cortex 47, 945-954. doi: 10.1016/j.cortex.2010.06.007

Perkell, J. S. (2012). Movement goals and feedback and feedforward control mechanisms in speech production. J. Neurolinguistics 25, 382-407. doi: 10.1016/j. jneuroling.2010.02.011

Pulvermüller, F., Huss, M., Kherif, F., Moscoso Del Prado Martin, F., Hauk, O., and Shtyrov, Y. (2006). Motor cortex maps articulatory features of speech sounds. Proc. Natl. Acad. Sci. U S A 103, 7865-7870. doi: 10.1073/pnas.05099 89103

Ridding, M. C., and Flavel, S. C. (2006). Induction of plasticity in the dominant and non-dominant motor cortices of humans. Exp. Brain Res. 171, 551-557. doi: 10. 1007/s00221-005-0309-2

Roy, A. C., Craighero, L., Fabbri-Destro, M., and Fadiga, L. (2008). Phonological and lexical motor facilitation during speech listening: a transcranial magnetic stimulation study. J. Physiol. Paris 102, 101-105. doi: 10.1016/j.jphysparis.2008. 03.006

Sato, M., Buccino, G., Gentilucci, M., and Cattaneo, L. (2010). On the tip of the tongue: modulation of the primary motor cortex during audiovisual speech perception. Speech Commun. 52, 533-541. doi: 10.1016/j.specom.2009. 12.004

Schecklmann, M., Volberg, G., Frank, G., Hadersdorfer, J., Steffens, T., Weisz, N., et al. (2011). Paired associative stimulation of the auditory system: a proof-of-principle study. PLoS One 6:e27088. doi: 10.1371/journal.pone.00 27088

Stefan, K., Kunesch, E., Benecke, R., Cohen, L. G., and Classen, J. (2002). Mechanisms of enhancement of human motor cortex excitability induced by interventional paired associative stimulation. J. Physiol. 543, 699-708. doi: 10. 1113/jphysiol.2002.023317

Stephan, K. E., Friston, K. J., and Frith, C. D. (2009). Dysconnection in schizophrenia: from abnormal synaptic plasticity to failures of self-monitoring. Schizophr. Bull. 35, 509-527. doi: 10.1093/schbul/sbn 176

Suppa, A., Li Voti, P., Rocchi, L., Papazachariadis, O., and Berardelli, A. (2013). Early visuomotor integration processes induce LTP/LTD-like plasticity in the human motor cortex. Cereb. Cortex doi: 10.1093/cercor/bht264. [Epub ahead of print]. 
Thabit, M. N., Ueki, Y., Koganemaru, S., Fawi, G., Fukuyama, H., and Mima, T. (2010). Movement-related cortical stimulation can induce human motor plasticity. J. Neurosci. 30, 11529-11536. doi: 10.1523/JNEUROSCI.1829-10.2010 Tourville, J. A., Reilly, K. J., and Guenther, F. H. (2008). Neural mechanisms underlying auditory feedback control of speech. Neuroimage 39, 1429-1443. doi: 10.1016/j.neuroimage.2007.09.054

Uy, J., Ridding, M. C., Hillier, S., Thompson, P. D., and Miles, T. S. (2003). Does induction of plastic change in motor cortex improve leg function after stroke? Neurology 61, 982-984. doi: 10.1212/01.wnl.0000078809.33581.1f

Watkins, K. E., Strafella, A. P., and Paus, T. (2003). Seeing and hearing speech excites the motor system involved in speech production. Neuropsychologia 41, 989-994. doi: 10.1016/s0028-3932(02)00316-0

Wolters, A., Schmidt, A., Schramm, A., Zeller, D., Naumann, M., Kunesch, E., et al. (2005). Timing-dependent plasticity in human primary somatosensory cortex. J. Physiol. 565, 1039-1052. doi: 10.1113/jphysiol.2005.084954
Conflict of Interest Statement: The authors declare that the research was conducted in the absence of any commercial or financial relationships that could be construed as a potential conflict of interest.

Received: 21 March 2014; accepted: 18 May 2014; published online: 03 June 2014. Citation: Sowman PF, Dueholm SS, Rasmussen JH and Mrachacz-Kersting N (2014) Induction of plasticity in the human motor cortex by pairing an auditory stimulus with TMS. Front. Hum. Neurosci. 8:398. doi: 10.3389/fnhum.2014.00398

This article was submitted to the journal Frontiers in Human Neuroscience.

Copyright $\odot 2014$ Sowman, Dueholm, Rasmussen and Mrachacz-Kersting. This is an open-access article distributed under the terms of the Creative Commons Attribution License (CC BY). The use, distribution or reproduction in other forums is permitted, provided the original author(s) or licensor are credited and that the original publication in this journal is cited, in accordance with accepted academic practice. No use, distribution or reproduction is permitted which does not comply with these terms. 